\title{
Optimal Design of Experiment for X-Ray Spectromicroscopy by Machine Learning
}

\author{
$\underline{\text { Tetsuro Ueno }}^{1, *}$, Hideitsu Hino $^{2}$, and Kanta Ono ${ }^{3}$
}

1. Quantum Beam Science Research Directorate, National Institutes for Quantum and Radiological Science and Technology, Sayo, Japan

2. The Institute of Statistical Mathematics, Tachikawa, Japan

3. Institute of Materials Structure Science, High Energy Accelerator Research Organization, Tsukuba, Japan

* Corresponding author, ueno.tetsuro@qst.go.jp

The total measurement time of an X-ray spectromicroscopy experiment using a scanning transmission X-ray microscope (STXM) is determined by a multiplication of a number of energy points $n_{\mathrm{e}}$, sample scanning points $n_{\mathrm{s}}$, and measurement time per each point $t_{\mathrm{m}}$ plus overhead. Overhead consists of time for data acquisition, moving of sample scanners, beamline optics and undulator properties (gap and phase of magnet arrays). An X-ray spectromicroscopy experiment with an STXM is performed as an image acquisition by sample scanning in an energy-by-energy regime. Moreover, moving of beamline optics such as a grating and mirrors takes longer time than that of piezoelectric actuators for sample scanning. Therefore, it is a good strategy to reduce $n_{\mathrm{e}}$ to reduce total measurement time. Another strategy to reduce total measurement time is an optimization of $t_{\mathrm{m}}$. One can reduce $t_{\mathrm{m}}$ at the expense of a signal-to-noise $(\mathrm{S} / \mathrm{N})$ ratio of spectra, which is proportional to $t_{\mathrm{m}}{ }^{1 / 2}$. It is important to reduce total measurement time without degrading the quality of spectra to extract physical or chemical parameters by analysis. Machine learning techniques are expected to resolve this issue. Ueno et al. proposed the adaptive design of an Xray magnetic circular dichroism (XMCD) spectroscopy experiment by Gaussian process (GP) modeling, a machine learning technique, and successfully reduced the total number of energy points to measure to evaluate magnetic moments with required accuracy [1].

In the present study, we performed GP modeling of X-ray absorption spectra (XAS) with variable number of energy points and $\mathrm{S} / \mathrm{N}$ ratios to assess optimal experimental conditions. Gaussian process modeling can predict a spectrum from a limited number of data points, and it is useful to find the optimal condition for X-ray absorption spectroscopy experiment. We used simulated XAS for GP modeling. X-ray absorption spectrum for $\mathrm{Sm}^{3+}$ was calculated using CTM4XAS software [2]. Gaussian noise was added to the noiseless spectrum to produce spectra with various $\mathrm{S} / \mathrm{N}$ ratio which is defined as a ratio between the intensity of the $M_{5}$ main peak of $\mathrm{Sm}^{3+}$ and standard deviation $\sigma$ of a Gaussian distribution. Gaussian process modeling was performed by $R$ software with the DiceKriging package [3].

Figure 1 shows the concept of the present study. First, a dataset of simulated XAS spectra with various $\mathrm{S} / \mathrm{N}$ ratios and number of data points is prepared. Next, GP modeling is applied to each XAS data and predicted XAS is calculated. Finally, a similarity (Pearson correlation coefficient) between a predicted spectrum and the reference spectrum (XAS with no noise and full data points) is evaluated.

Figure 2 shows the results for GP modeling, and the similarity between GP-predicted spectra and the reference spectrum. As an overall trend, similarity values for high data points density and high $\mathrm{S} / \mathrm{N}$ ratio are close to 1 . Thus, the accuracy of spectrum prediction increases in such conditions. Similarity values do not exceed 0.8 for very low $\mathrm{S} / \mathrm{N}$ ratio $(\mathrm{S} / \mathrm{N}=5)$. Therefore, XAS spectra with poor $\mathrm{S} / \mathrm{N}$ ratio fail to retrieve the predicted spectrum with high similarity in spite of increasing data points. On the other hand, 
high similarity values above 0.9 are observed for $\mathrm{S} / \mathrm{N} \geq 50$ with at least 30 energy points. Similarity values reach almost 1 for $\mathrm{S} / \mathrm{N} \geq 100$ with high data points density $\left(n_{\mathrm{e}} \geq 100\right)$. That means $\mathrm{S} / \mathrm{N}=100$ is enough in this case. For example, we can expect four-times-faster measurement for same $n_{\mathrm{e}}$ to obtain same Pearson correlation coefficient, if we set $\mathrm{S} / \mathrm{N}=100$ rather than $\mathrm{S} / \mathrm{N}=200$.

In conclusion, we examined GP modeling of XAS with various $\mathrm{S} / \mathrm{N}$ and $n_{\mathrm{e}}$ to find optimal experimental condition. Necessary and sufficient experimental conditions can be obtained by setting energy point density and S/N ratio depending on expected spectrum structures. Moreover, real-time GP modeling during measurement can automatically find optimal experimental conditions [4].

\section{References:}

[1] T Ueno et al., npj Comput. Mater. 4 (2018) 4.

[2] E Stavitski and F M F de Groot, Micron 41 (2010) 687.

[3] O Roustant, D Ginsbourger, and Y Deville, J. Stat. Softw. 51 (2012) 1.

[4] HH acknowledges the support from CREST (No. JPMJCR1761) from Japan Science and Technology Agency (JST).
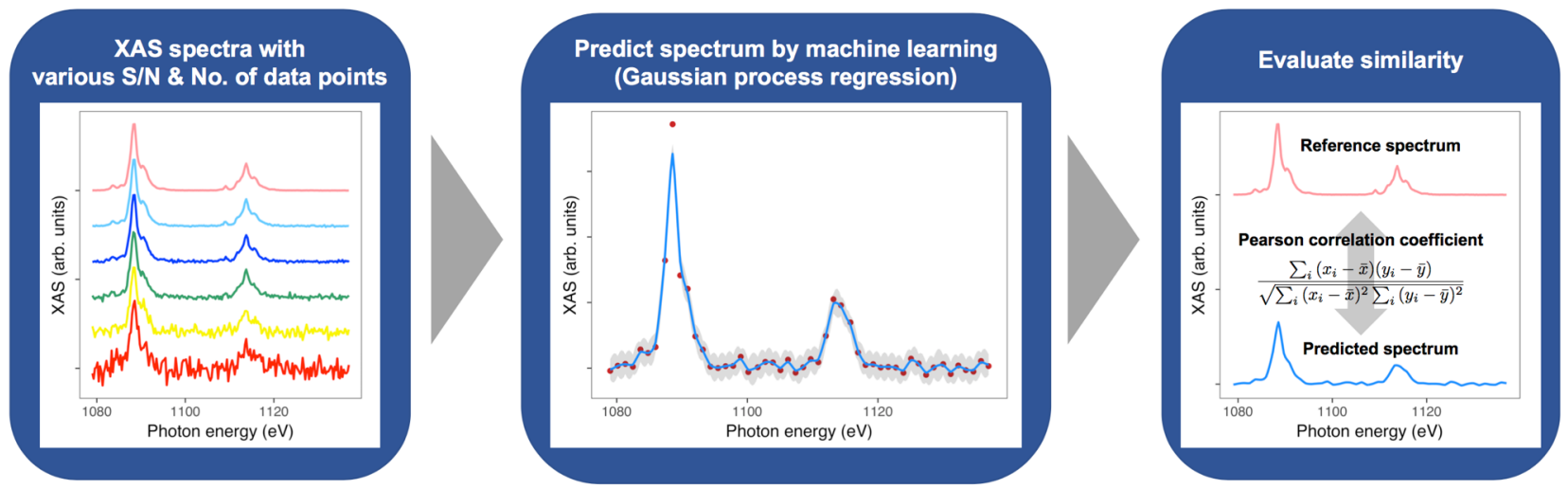

Figure. 1. Schematic of the concept of the present study.

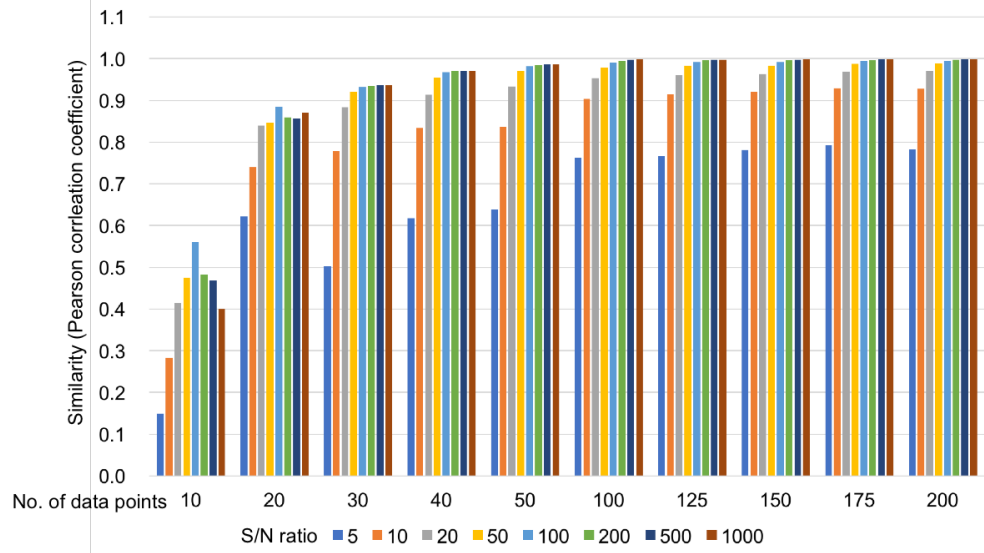

Figure. 2. Similarity values between GP-predicted spectra and the reference spectrum. 